\title{
How do you measure "success" in a biotechnology company?
}

\section{The impact of the institutional investor on a company's future is often overlooked.}

\author{
Elizabeth M. Greetham
}

No matter where you are in creating or developing a biotechnology company, you will undoubtedly have immediate and long-term goals by which to measure your company's success. As your company grows, these goals will vary: Whether it is securing venture capital funding, entering into a collaboration with a partner, floating a successful initial public offering or secondary issue, having a product candidate complete a successful clinical trial, or actually launching a product on the market, the scale by which you measure your company will change.

One measure of success that is often overlooked by beginning biotechnology companies is the purchase of large blocks of stock by an institutional investment fund. These funds most often use pension funds, or in some cases limited partnership money, to invest in publicly traded and emerging biotechnology companies ${ }^{1}$.

Beyond the fact that they can often deliver sums in excess of $\$ 20$ million into a biotechnology company's bank account, many times they are viewed by the financial community as validators of a company's product or platform technology. This can help attract other investors in your company at transition points when funding may be critical-for example, moving into phase III clinical trials, or launching a new product. For these reasons, it is important from the outset that you launch your biotechnology company with the full realization of what "success" means from the institutional investor's point of view as a way of strategizing your business development.

\section{The competitive landscape}

In trying to pick which biotechnology companies to invest in, how does an institutional investor measure your potential for success? To begin with, the principal gatekeeper behind our investment decisions is "our business model" based on the pharmaceutical industry. We measure every biotechnology company as a business participating in drug development and want to see the $25 \%$

Elizabeth M. Greetham is a portfolio manager at Weiss Peck \& Greer, One New York Plaza, 30th Floor, New York, NY 10004.
Biotechnology examples are numerous. The simple

lesson from all this is to know that your business is drug development and that the methodical approach of putting one foot in front of the other carries the day.

operating margin achieved by the pharmaceutical industry.

To pick out companies with this potential, we survey the competitive environment. On the basis of pharmaceutical data, about one in five of the products in clinical trials could actually reach the market. However, with biotechnology companies, there are two other biotechnology-specific risk factors that must be considered in analyzing the potential success rate.

The first is the industry's focus on therapeutic voids as initial targets. These largely multifactorial diseases such as amyotrophic lateral sclerosis (ALS), adult respiratory distress syndrome (ARDS), septic shock, wound healing, and psoriasis have no existing products for a reason: They are extremely difficult to treat. This means that it will be extremely costly and difficult to generate convincing clinical data that will meet with the US Food and Drug Administration's (Rockville, MD) approval.

Beyond that, the "one product nature" of the majority of biotechnology companies means that the company's chances are literally hit or miss: They will either prosper or fail based on the results of their clinical trials. For these reasons, the biotechnology success rate is approximately one in eight or one in nine. Notwithstanding this relatively low success factor, for the investor there is a substantial opportunity for superior capital appreciation over time.

Success will be determined largely by the ability to allocate and control research and development risk. Our formula for success is that we invest $65-75 \%$ of the fund's assets in companies in late-stage product develop- ment: Either their product is in phase III, a new drug application has been filed with the FDA, or the company has recently brought a product to market. Another $10-25 \%$ of the fund is invested in companies with products in phase I/II clinical trials that look promising based on preclinical data that validates the basic biology. The remaining $10-15 \%$ of the fund is invested in "enabling technology" companies-basic-research companies whose platform technology promises to transform some aspect of drug discovery and/or development. These companies must be able to generate repeat revenues and have "true" economies of scale.

\section{Judging product risk}

Since we invest nearly $85 \%$ of the fund's assets in product companies, if you are a biotechnology company trying to make decisions about which therapeutic areas to enter, it is important that you understand how we rate a product's therapeutic potential. Unlike the initial focus of the biotechnology industry, we tend to avoid pioneering therapeutics in new disease areas It was a conscious decision to avoid septic shock and ARDS, as these diseases were multifactorial and had uncertain endpoints-in the case of the FDA, ARDS, for example, it was initially mortality, then reduction in ventilator days, and then back again to mortality as the endpoint. In short, it is too easy for the FDA to move the goal posts to justify the risk in these disease areas.

Rather, we tend to fund "known diseases" that have clearly defined regulatory pathways but for which current treatments are inadequate. For example, new product candidates that treat cancer, diabetes, Parkinson's disease, or stroke are actively sought out if they are not merely "me-too" products in an already overcrowded niche. To differentiate themselves they must be innovative, have clear therapeutic superiority, and demonstrate cost effectiveness.

Our second criteria is the clinical viability of the drug candidate. Perhaps the most frequently broken rule of drug development in biotechnology companies is that of the necessity of having a clear understanding of "the label" sought for a product at the earliest possible point in the development process. 
Without this information, it is difficult, if not impossible, to design trials that answer the efficacy and statistical questions to the satisfaction of the FDA. Moreover, without this information, a realistic patient population cannot be identified, making competitive product positioning impossible. This obscures any realistic appreciation of a product's potential, confuses management about how to allocate scarce resources for product development, and scares off a potential corporate partner.

What about products in the phase I/II category for which the clinical data is not yet in? The first step here is to validate the science. For example, the biological need for the GPIIb/IIIa inhibitors to bind greater than $80 \%$ of platelets to achieve a sufficient blockade and hence efficacy has been established by Centocor (Malvern, PA), COR Therapeutics (S. San Francisco, CA), and possibly Merck (Whitehouse Station, NJ) through their clinical programs. A second example involves the need for a drug candidate to work in animal models-whether or not the specific pathway has been validated. Guilford Pharmaceutical's (Baltimore, MD) positive primate data establishing the regenerative properties of their lead neuroimmunophilins in Parkinson's disease is a classic example.

In this area of clinical development, Aesop's fable of "the tortoise and the hare" is an apt example of strategies that produce success and those that end in failure. The hare's excessive confidence led him down a path that involved a "quick fix." Biotechnology examples are numerous. The simple lesson from all this is to know that your business is drug development and that the methodical approach of putting one foot in front of the other carries the day.

\section{Tool technologies}

Finally, what about the tool technologies? Although they represent a minor part of our portfolios, their significant initial successes in the market recommend them for serious consideration. Tool technologies must be judged initially on their ability to meet their customer's needs. For biotechnology, the customer is the pharmaceutical industry, and to the degree that the tool is able to industrialize basic research, it is valuable. Genomics, combinatorial chemistry, highthroughput screening, and proteomics are all examples of tools that the pharmaceutical customer perceives to be vital to their continued growth.

Tool companies, in our opinion, are differentiated by their management teams. One values the management in its ability to close deals on highly favorable terms for itself with its customers. The basic strategy to accomplish this is to create "repeat-revenue" sales.
In this regard, the managements of both Incyte (Palo Alto, CA) and Millennium (Cambridge, MA) deserve high marks. Initially, most observers scoffed at the concept that a "Microsoft" for the pharmaceutical industry could be created. Incyte's "database model" for selling information tools to pharmaceutical companies not only proved that this is a possibility but soon became an industry-wide business model mantra.

A key element to Incyte's continued success in its \$160 million plus already invested in technologies that will maintain its leadership in this area. The outstanding question remains whether investors ${ }^{1}$ needs for earnings growth will detract from Incyte's lead position with this scale of R\&D investment.

Millennium's management has taken a different tack by developing a leveraged business model to maximize cash flow and nearterm revenues while retaining significant downstream rights. The company has taken its core gene-discovery platform and repackaged it for various consumers. The small molecule platform belongs to Millennium, which is funded by major pharmaceutical partners. Millennium BioTherapeutics, funded by Eli Lilly (Indianapolis, IN), searches for protein and peptide drugs; Millennium Plant Agriculture, funded by Monsanto (St. Louis, MO) applies this technology to agricultural biotechnology.

From an investors standpoint, the jury is still out on whether this business strategy will deliver long-term profits. I believe there will be many "hares" strategies built on corporate partnerships that are short-term band-aids, as has been demonstrated by some of the combinatorial chemistry companies. Success will equate with becoming an integral part of the discovery engine of the pharmaceutical company and taking the tortoise-like role of slowly but surely adding value.

\section{Separating winners from losers}

The three basic resources any company has to add value are research, manufacturing, and marketing. For most biotechnology companies, research is the only viable focus because they do not have sufficient cash to initiate manufacturing and marketing. Amgen (Thousand Oaks, CA) stands out as the exception to the rule, as it was able to create two back-to-back blockbuster drugs and thus fully exploit all three revenue resources. All the other major biotechnology companies have required the outsourcing of at least one of these variables. For example, Chiron (Emeryville, CA) and Genentech (S. San Francisco, CA) have major pharmaceutical partners supporting their R\&D effort, Genzyme (Cambridge, MA) has created multiple spinoffs and relies on Wall Street to fund the R\&D of each of these, Biogen (Cambridge,
MA) and Centocor both out-licensed their original technologies in order to use the royalty stream to fund their internal research.

Of these models for funding research, the outlicensing of the first product has become the most popular. A word of warning is necessary here. Many times management tries to convince itself that because there is a lower risk in taking this route, a lower operating return would suffice as well. In my opinion, royalties or operating profits in the low to mid teens are insufficient for long-term survival and merely represent short-term ways to pay the near-term bills. Given the popularity of this model, a valid question is, "What is the alternative?"

This is where management must identify their company's "differentiated value-added skills" and find the means to raise sufficient funds to maximize the successful commercialization of these resources. Alkermes (Cambridge, MA) is one example of a company that chose to mortgage its firstborn, but then went on to find a way to generate the profitability it needs for long-term survival.

After deciding to mortgage their first product, RMP-7, management identified a second auxiliary technology, ProLease, that was part of their initial R\&D effort. Their strategy was to set up manufacturing for this technology and sell it at an appropriate margin to other drug developers. Alkermes' worldwide agreement to manufacture human growth hormone in a ProLease formulation for Genentech demonstrates the first fruits of this decision. Most importantly, this strategy has paid off by clearing the hurdle for profitability by which we judge all biotechnology investments.

\section{Conclusions}

In its recent history, biotechnology companies have been a bit short-sighted in calculating their definition of success. The low-double-digit, R\&D funding milestone deal may pay the bills in the short term, but does not allow for reinvesting in $\mathrm{R} \& \mathrm{D}$. The rush to phase III to create shareholder value is akin to a "teeter-totter," not success.

For the industry as a whole, failure to move beyond the IPO and create real value through products that impact healthcare can only result in the drying up of the funding well. Investors need sustainable, rapid profit growth. As you build your biotechnology company, keeping your eye on that goal is perhaps the best way to assure its "success."

1. The Weiss Peck \& Greer Life Science Funds were established in 1992. They primarily invest in publicly traded biotechnology companies. In essence, the Life Science Funds are a business whose management capitalizes on their value added skills: clinical development and product commercialization. 